\title{
A Study of Malaria Epidemic Model Using the Effect of Lost Immunity
}

\author{
A. S. Talawar ${ }^{1}$, U. R. Aundhakar ${ }^{2}$ \\ Department of Statistics, Karnatak University, Dharwad Karnataka State, India
}

\begin{abstract}
The model is developed to apply malaria data for both human population (hosts) and vector (mosquito) population. The hosts are divided into four compartments, susceptible $S_{h}(t)$, infected $I_{h}(t)$ infectious $Z_{h}(t)$ and removed $R_{h}(t)$ at time $t$ and vectors are divided into three compartments susceptible mosquitoes $S_{m}(t)$, infected mosquitoes $I_{m}(t)$ and infectious mosquitoes $Z_{m}(t)$ at time $t$. Recovered group for mosquitoes is not considered, because mosquitoes are assumed to remain infectious until death. As the model has two different populations (hosts and vectors), the expected basic reproduction number reflects the infection transmitted from human to vector and vice-versa. In the present study, we have considered the effect of lost immunity and the partially recovered rate as a function of infection dependent. We have studied the application of optimal control theory to the model. Numerical illustration of the model is also given.
\end{abstract}

Keywords: recurrent epidemic, basic reproduction number, immunity and optimal control.

\section{Introduction}

According to the latest WHO estimates, released in September 2015, there were 214 million cases of malaria in 2015 and 438000 deaths. Between 2000 and 2015, malaria incidence fell by $37 \%$ globally; during the same period, malaria mortality rates decreased by $60 \%$. An estimated 6.2 million malaria deaths have been averted globally since 2000. Sub-Saharan Africa continues to carry a disproportionately high share of the global malaria burden. In 2015 , the region was home to $89 \%$ of malaria cases and $91 \%$ of malaria deaths. In 2013, an estimated 437000 African children died before their fifth birthday due to malaria. Globally, the disease caused an estimated 453000 under-five deaths in 2013

Malaria is caused by four members of the genus Plasmodium; Plasmodium vivax ( $\mathrm{P}$. vivax), Plasmodium falciparum (P. falciparum), Plasmodium malariae ( $\mathrm{P}$. malariae) and Plasmodium ovale (P. ovale). Plasmodium falciparum (malignant tertian malaria) and P. malariae (quartan malaria) are the most common species of malarial parasite in Asia and Africa. Plasmodium vivax (benign tertian malaria) predominates in Latin America, India and Pakistan. Plasmodium ovale (ovale tertian malaria) is almost exclusively found in Africa.

In most cases, malaria is transmitted through the bites of female Anopheles mosquitoes. The intensity of transmission depends on factors related to the parasite, the vector, the human host, and the environment. Transmission is more intense in places where the mosquito lifespan is longer (so that the parasite has time to complete its development inside the mosquito) and where it prefers to bite humans rather than other animals. Transmission also depends on climatic conditions that may affect the number and survival of mosquitoes, such as rainfall patterns, temperature and humidity (Talawar and Pujar, 2011). In many places, transmission is seasonal, with the peak during and just after the rainy season. Malaria epidemics can occur when climate and other conditions suddenly favour transmission in areas where people have little or no immunity to malaria. They can also occur when people with low immunity move into areas with intense malaria transmission, for instance to find work, or as refugees. Human immunity is another important factor, especially among adults in areas of moderate or intense transmission conditions. Partial immunity is developed over years of exposure, and while it never provides complete protection, it does reduce the risk that malaria infection will cause severe disease. For this reason, in areas with less transmission and low immunity, all age groups are at risk.

The first deterministic compartmental model was developed by Ross (1915), where host and vector populations are modeled as susceptible-infected-susceptible (SIS) and susceptible-infected (SI) respectively. Macdonald (1957) developed the malaria model by modifying the Ross model adding an exposed class. Smith et al.,(2012), based on a model of Ross and Macdonald, concluded that there is a relationship between the ratio of mosquitoes to humans and the number of infected humans, hence it is not necessary to kill every mosquito to end disease transmission. Mwanga et al (2014a) developed SIR malaria model for human population and SI model for mosquito population and used different parameter values estimated by Agusto et al (2012), Chitnis et al (2006) and Mukandavire et al (2010) for malaria transmission model. They studied the effect of infective immigrants in the presence drug resistance strains. Mwanga et al (2014b) also studied the malaria model in the presence parameter uncertainty using MCMC method. A person infected with malaria may be reinfected before recovering completely if bitten again by an infectious mosquito (Nedelman, 1985). Therefore, in the present paper we consider partially recovered humans (removals) with temporary immunity and lost immunity. We develop SEIR for human (hosts) population and SEI for mosquito (vector) population. We study the effect of lost immunity on state variables.

\section{Formulation of the Model}

The model divides the human population into four groups or compartments; those who are susceptible to the disease,

\section{Volume 4 Issue 12, December 2015}




\section{International Journal of Science and Research (IJSR) \\ ISSN (Online): 2319-7064}

Index Copernicus Value (2013): 6.14 | Impact Factor (2014): 5.611

those who are infected but not infectious, those who are

Following is the transfer diagram of the malaria disease infectious and those who are in some form of recovery.

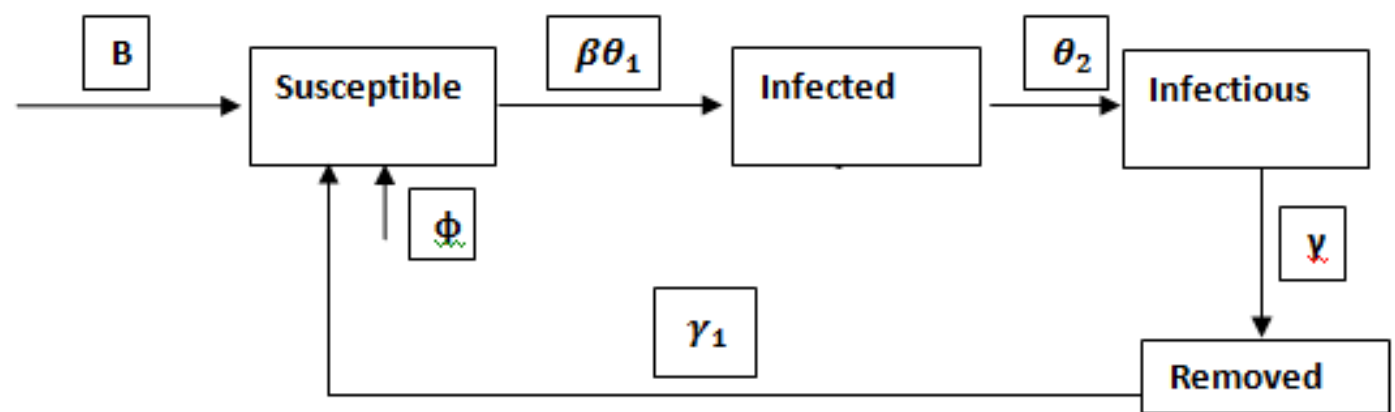

The system of differential equations is

$$
\begin{gathered}
S^{s}=B-\left(\beta \theta_{1}\left(\frac{f}{f+v}\right)-\phi\right) s+\gamma_{1} R \\
I^{s}=\left(\beta \theta_{1}\left(\frac{f}{f+v}\right)\right) S-\left(\theta_{2}+\mu\right) I(1)
\end{gathered}
$$

$Z^{s}=\left(\theta_{2}+\mu\right) I-\gamma Z$

$R^{s}=\gamma Z-\gamma_{1} R$

Where $N=S+I+Z+R, \quad B$ is the recruitment of susceptible, $\beta$ is the contact rate between bacterial and susceptible hosts, $\theta_{1}$ is the proportion of infected individuals who are infectious, $\theta_{2}$ is the proportion of infected mosquitoes who are also infectious, $\mu$ is immigration of infectious individuals, $\phi$ rate at which susceptible remain uninfected even after contract, $\gamma$ rate at which individuals acquired immunity and $\gamma_{1}$ rate at which removed individuals lose their immunity.

Due to biological reasons, only non-negative solutions of the system (1) are acceptable. It is necessary to study the solution properties of the system (1) in the closed set,

$$
\Gamma=\left\{(S, I, Z, R) \in R^{4}, S+I+Z+R \leq N\right\} \text { (2) }
$$

A vector (four tuple), $E=(S, I, Z, R)$ is said to be an equilibrium point for the system (1) if it satisfies the following conditions,

$$
\mathrm{S}^{\prime}=0, \mathrm{I}^{\prime}=0, \mathrm{Z}^{\prime}=0 \text { and } \mathrm{R}^{\prime}=0 \text { (3) }
$$

An equilibrium point $E$ is meaningful iff $E \in \Gamma$. The equilibrium points are said to be disease free or endemic depending on $I$ and $Z$. If there is no disease $(I=0$ and $Z=0$ ), then the equilibrium point is said to be a diseasefree equilibrium (DFE) point, otherwise if $I \neq 0$ or $Z \neq 0$ ( in other words $I>0$ or $Z>0$ ), then the equilibrium point is called endemic.

Theorem: The system (1) admits at most two equilibrium points; one disease free equilibrium point $\left(P_{0}\right)$ and one endemic equilibrium point $\left(P^{*}\right)$, where $P_{0}=\left(\frac{B}{\phi}, 0,0,0\right)$ and $P^{*}=\left(S^{*}{ }_{x} I^{*} Z^{*}{ }_{x} R^{*}\right)$. The state variables at endemic equilibrium point $P^{*}$ are

$$
\begin{aligned}
& S^{*}=\frac{\frac{B}{\phi}}{\left(\frac{1}{\theta_{2}+\mu}+\frac{1}{\gamma}+\frac{1}{\gamma_{1}}+1\right)^{*}} \\
& I^{*}=\frac{\frac{1}{\theta_{2}+\mu}}{\left(\frac{1}{\theta_{2}+\mu}+\frac{1}{\gamma}+\frac{1}{\gamma_{1}}+1\right)}, \\
& Z^{*}=\frac{\frac{1}{\gamma}}{\left(\frac{1}{\theta_{2}+\mu}+\frac{1}{\gamma}+\frac{1}{\gamma_{1}}+1\right)^{*}}, R^{*}=\frac{1 / \gamma_{1}}{\left(\frac{1}{\theta_{2}+\mu}+\frac{1}{\gamma}+\frac{1}{\gamma_{1}}+1\right)}
\end{aligned}
$$

Threshold values: There are three commonly used threshold values in epidemiology the basic reproduction number $\left(R_{0}\right)$, the contact number $(\sigma)$ and the replacement number $(R)$. The basic reproduction number is defined as the average number of secondary infections that occurs when one infective is introduced into a completely susceptible population. These are all at the beginning of the spreading of an infection disease; the entire population (except the infective invader) is susceptible. The $R_{0}$ is defined at the time of invasion, where as $\sigma$ and $\mathrm{R}$ are defined at all times.

Due to differences in demographic rates, rural-urban gradients and contact structure, different human populations may be associated with different values of $R_{0}$ for the same disease ( Hethcote, 2000 ; Anderson and May, 2005).

Proposition: The $\Gamma$ is positively invariant under the flow reduced by the system of differential equations (1).

Proof: The system of differential equations (1) can be written in the following way;

$$
\frac{d X}{d t}=M(X) X+F(5)
$$

Where $\mathrm{E}=(\mathrm{S}, \mathrm{I}, \mathrm{Z}, \mathrm{R}), \mathrm{F}=(B, 0,0,0)$, and

$$
M(X)=\left[\begin{array}{ccc}
\beta \theta_{1}\left(\frac{f}{f+v}\right)-\phi & 0 & 0 \gamma_{1} \\
\beta \theta_{1}\left(\frac{f}{f+\nu}\right) & -\left(\theta_{2}+\mu\right) & 00 \\
0 & \left(\theta_{2}+\mu\right) & -\gamma 0 \\
& 00 \gamma-\gamma_{1} &
\end{array}\right]
$$




\section{International Journal of Science and Research (IJSR) \\ ISSN (Online): 2319-7064 \\ Index Copernicus Value (2013): 6.14 | Impact Factor (2014): 5.611}

As $M(X)$ has all off-diagonal entries non-negative, $M(X)$ is Metzler matrix. Using the fact that $F \geq 0$, the system (1) is positively invariant in $R_{+}^{4}$. Which means that any trajectory of the system of differential equations starting from an initial state in the positive orthant $R_{+}^{4}$ remains forever in $R_{+}^{4}$.

\section{Time dependent immunity (TDI)}

We consider time dependent immunity acquisition rate, $\gamma=\gamma(t)$. Assume that immunity is initially nil and that immunity tends asymptotically to a limiting value, say $\Lambda$.
The rate of immunity $(\gamma)$ is replaced by $\Lambda\left(1-e^{-v t^{2}}\right)$. Then the last two equations of the system (1) of differential equations can be written as,

$$
\begin{aligned}
& Z^{s}=\left(\theta_{2}+\mu\right) I-\mathrm{A}\left(1-e^{-v t^{2}}\right) Z \\
& R^{s}=\mathrm{A}\left(1-e^{-v t^{2}}\right) Z-\gamma_{1} R \text { (6) }
\end{aligned}
$$

The modification has no effect on stationary values, since $y \rightarrow \mathrm{A}$ as $\mathrm{t} \rightarrow \infty$. So the stationary point remains the same only with $A$ replacing $\gamma$ in system (1).

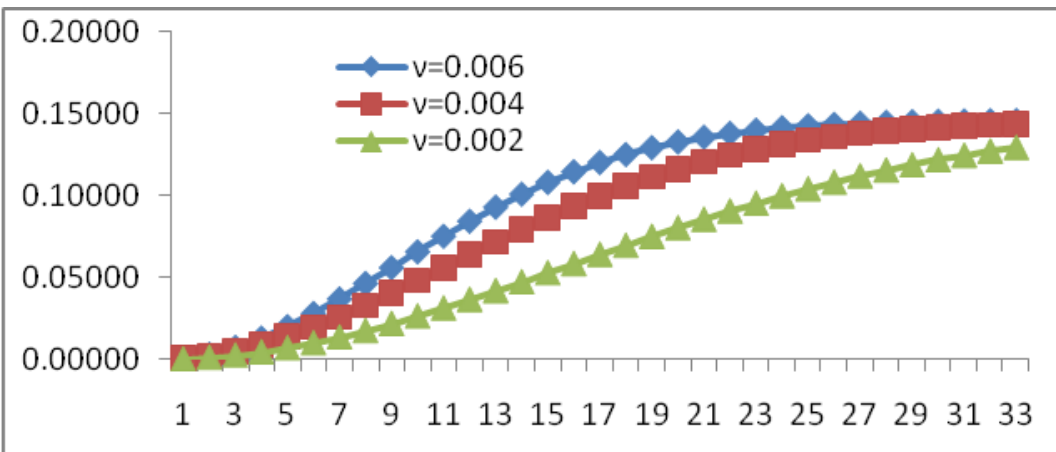

Figure 1: Effect of vaccination on time dependent immunity v/s Age

Note that this time-dependent model preserves the crossover phenomenon and the urban versus rural phenomenon.

\section{Application of the Model}

The model (1) is reformulated to apply to malaria data for both human population (hosts) as well as to vector population. The hosts are divided into four compartments as before, susceptible, $S_{\hbar}(t)$, infected, $I_{h}(t)$, infectious , $Z_{\hbar}(t)$ and removed, $R_{h}(t)$ at time $\mathrm{t}$ and vectors are divided into three compartments susceptible mosquitoes, $S_{m}(t)$, infected mosquitoes, $I_{m}(t)$ and infectious mosquitoes, $Z_{m}(t)$ at time t. Recovered group for mosquitoes is not considered, because mosquitoes are assumed to remain infectious until death. The system differential equations for human population is

$S_{h}^{s}=B_{h}-\left(\beta_{h m} \theta_{1} I_{m}-\phi_{h}\right) S_{h}+\gamma_{1} R_{h}$

$$
I_{h}=\left(\beta_{h m} \theta_{1} I_{m}\right) S_{h}-\left(\theta_{2}+\mu_{h}\right) I_{h}(7)
$$

$Z_{\hbar}{ }^{*}=\left(\theta_{2}+\mu_{h}\right) I_{h}-\gamma_{h} Z_{\hbar}$

$R_{h}^{s}=\gamma_{h} Z_{h}-\gamma_{1 h} R_{h}$

The system differential equations for mosquito population is $S_{m}{ }^{s}=B_{m}-\left(\beta_{m h} \theta_{1} I_{h}\right) S_{m}$

$$
I_{m}^{\prime}=\left(\beta_{m h} \theta_{1} I_{h}\right) S_{m}-\left(\theta_{2}\right) I_{m}(8)
$$

$Z_{m}^{\prime}=\left(\theta_{2}\right) I_{m}-\gamma_{m} Z_{m}$

The model has two different populations (hosts and vectors) and therefore the expected basic reproduction number reflects the infection transmitted from human population to vector and vice-versa. Thus the basic reproduction number, $R_{0}$ can be taken as $\sqrt{R_{h m} X R_{m h}}$. Where $R_{h m}$ basic reproduction number for the infection from hosts to vectors, $R_{h m}=\frac{B_{h} \beta_{h m} \theta_{1}}{\phi_{h}\left(\theta_{2}+p_{h}\right)}$ and basic reproduction number for the infection from vectors to hosts, $R_{m h}=\frac{B_{m} \beta_{m h} \theta_{1}}{\left(\theta_{2}\right)}$.

$$
\text { Thus, } R_{0}=\sqrt{\frac{B_{h} \beta_{h m m} \theta_{1}^{2}}{\phi_{h}\left(\theta_{2}+\mu_{h}\right)} \frac{B_{m} \beta_{m h}}{\left(\theta_{2}\right)}}
$$

\section{4 (a). Effect of lost immunity}

The quantity $\gamma_{1} R$ in system (1) allows for a return path from the partially recovered or losing immunity, back to the susceptible compartment. Furthermore, $\gamma_{1}$ is taken as a function of $\beta_{\mathrm{hm}} \theta_{1}$ (that is infection dependent recovery rate) in difference to the observation by many that the greater the endemicity of the disease, the greater the immunity among population. Thus $\gamma_{1}$ should decrease with increasing $\beta_{h m} \theta_{1}$. The exact relationship is in terms of a parameter $\tau$ and takes the form $\gamma_{1}\left(\beta_{h m} \theta_{1}\right)=\frac{\beta_{h m m} \theta_{1} e^{-\theta_{h k m} \theta_{1} \tau}}{1-e^{-\beta_{h m} \theta_{1 \tau}}}$ (Aron and May, 1982) 


\section{International Journal of Science and Research (IJSR) \\ ISSN (Online): 2319-7064}

Index Copernicus Value (2013): 6.14 | Impact Factor (2014): 5.611

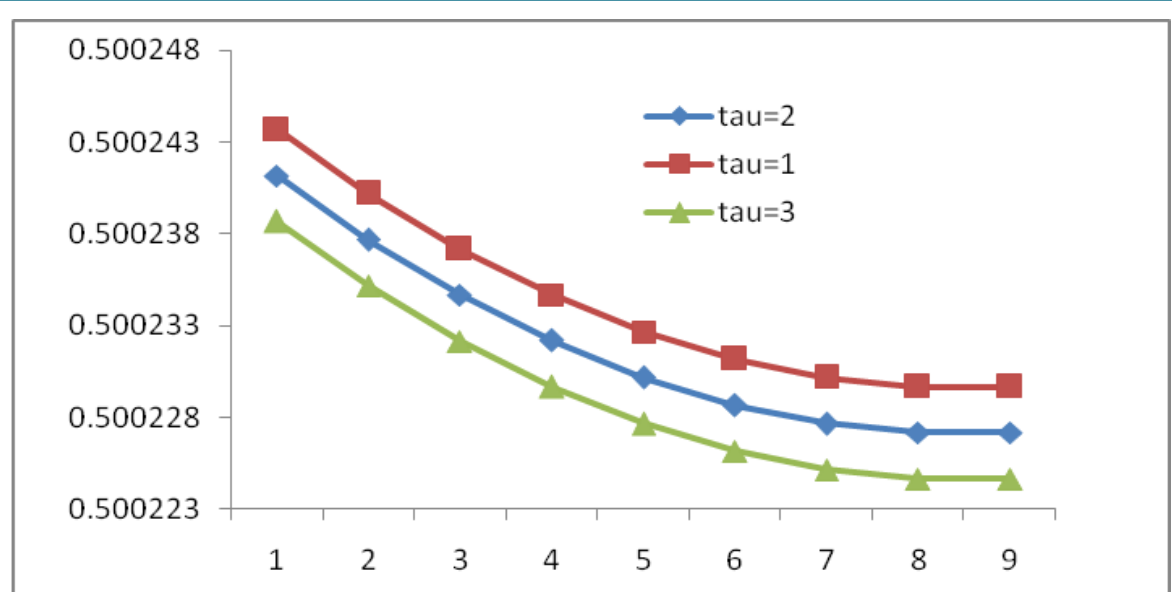

Figure 2: Effect of lost immunity as a function of infection rate for various $\tau$

$$
(\tau=1, \tau=2, \tau=3 \text { ) }
$$

\section{Application of Optimal Control Theory}

For analysis of the optimal level of effort required to control the spread of malaria, two control measures are taken into consideration. Screening and treatment of asymptomatic infective individuals $\left(u_{1}\right)$ and treatment of symptomatic individuals with antimalarial drugs $\left(u_{2}\right)$. The optimal control problem is stated as follows;

Minimize the number of infected (both asymptomatic, $I$ and symptomatic, $Z$ ) within the time horizon $T$, that is $t \in[0, T]$ given by the function

$$
\begin{aligned}
& \quad l=\min _{\mathrm{u} \in U} \int_{0}^{T} F(t, u, q)+h(t, u) d t \\
& \qquad=\left\{u_{j} \text { given that } u_{j}(t)\right. \text { is Lebesgue m } \\
& \text { Where } \\
& \quad F(t, u, q)=A_{12} I_{\hbar}+A_{2} Z_{\hbar}+c_{1} u_{1}\left(I_{h}\right)+c_{2} u_{2}\left(Z_{\hbar}\right) \\
& h(t, u)=\frac{1}{2} w_{1} u_{1}{ }^{2}(t)+\frac{1}{2} w_{2} u_{2}{ }^{2}(t)(14)
\end{aligned}
$$$$
U=\left\{u_{j} \text { given that } u_{j}(t) \text { is Lebesgue measurable } 0 \leq u_{j}(t) \leq 1 \text {, for } j=1,2, t \in[0, t]\right\}
$$

Subject to the state system of equations (7-8),

$S_{h}=B_{h}-\left(\beta_{h m} \theta_{1} I_{m}-\phi_{h}\right) S_{h}+\gamma_{1 h} R_{h}$

$I_{h}^{s}=\left(\beta_{h m} \theta_{1} I_{m}\right) S_{h}-\left(\theta_{2}+\mu_{h}+u_{1}\right) I_{h}$

$Z_{\hat{h}}=\left(\theta_{2}+\mu_{h}\right) I_{h}-\left(\gamma_{h}+u_{2}\right) Z_{h}$

$R_{h}{ }^{s}=\gamma_{h} Z_{\hbar}-\gamma_{1 h} R_{h}+u_{1} I_{h}+u_{2} Z_{h}$ (11)

$S_{m}^{s}=B_{m}-\left(\beta_{m h} \theta_{1} I_{n}\right) S_{m}$

$I_{\mathrm{m}}=\left(\beta_{\mathrm{mh}} \theta_{1} I_{\mathrm{h}}\right) S_{\mathrm{m}}-\left(\theta_{2}\right) I_{\mathrm{m}}$

$Z_{\mathrm{m}}^{s}=\left(\theta_{2}\right) I_{\mathrm{m}}-\gamma_{\mathrm{m}} Z_{\mathrm{m}}$

and the control constraint
Where

and $\mathrm{q}$ is the solution of the state system (7). $c_{1}$ and $c_{2}$ are the individual costs for screening and treatment of asymptomatic individuals and for the treatment of symptomatic individuals respectively, $A_{1}, A_{2}, w_{1}$ and $w_{2}$ are

$$
l=\int_{0}^{T}\left[A_{1} I_{h}+A_{2} Z_{\hbar}+c_{1} u_{1}\left(I_{h}\right)+c_{2} u_{2}\left(Z_{h}\right)+\frac{1}{2} w_{1} u_{1}{ }^{2}(t)+\frac{1}{2} w_{2} u_{2}{ }^{2}(t)\right] d t
$$

This performance specification involves the number of asymptomatic and symptomatic individuals as well as effect of implementing the asymptomatic control $\left(u_{1}\right)$ and symptomatic control $\left(u_{2}\right)$.

We find optimal control pair $\left(u_{1}^{*}, u_{2}^{*}\right)$ such that

$$
f\left(u_{1}^{*}, u_{2}^{*}\right)=\operatorname{Min}\left\{j\left(u_{1}, u_{2}\right) ;\left(u_{1}, u_{2}\right) \in U\right\}
$$

Now applying the necessary condition from Pontryagin's Maximum Principle (PMP), we have the problem the relative weights. $F(t, u, q)$ defines actual costs while $h(t, u)$ defines the background costs (such as ordering, shipment and distribution, and storage).

Therefore the optimal control problem (10) is to minimize the cost function

minimizing a Hamiltonian, $\mathrm{H}$ pointwisely with respect to $u_{1}$ and $u_{2}$

Theorem: Given an optimal control pair $U^{*}(t)=\left(u_{1}^{*}(t), u_{2}^{*}(t)\right)$ and a solution of the system (11) there exists adjoint variables $\lambda_{S_{h}}(t), \lambda_{I_{h}}(t), \lambda_{z_{h}}(t), \lambda_{R_{h}}(t), \lambda_{S_{m}}(t), \lambda_{I_{m}}(t)$ and $\lambda_{z_{m}}(t)$ such that, the Hamiltonian is given by 


\section{International Journal of Science and Research (IJSR) \\ ISSN (Online): 2319-7064}

Index Copernicus Value (2013): 6.14 | Impact Factor (2014): 5.611

$$
\begin{aligned}
& H\left(S_{\mathfrak{h}}, I_{\mathfrak{h}}, Z_{\mathfrak{h}}, R_{h}, S_{m}, l_{m}, Z_{m}\right)=\left[\left[A_{1} I_{h}+A_{2} Z_{\hbar}+c_{1} u_{1}\left(a_{h}\right)+c_{2} u_{2}\left(Z_{\hbar}\right)+\frac{1}{2} w_{1} u_{1}^{2}(t)+\frac{1}{2} w_{2} u_{2}^{2}(t)\right]+\lambda_{S_{h}}\left(B_{h}-\right.\right. \\
& \left.\left(\beta_{h m} \theta_{1} I_{m}-\phi_{h}\right) S_{h}+\gamma_{1 h} R_{h}\right)+\lambda_{I_{h}}\left(\left(\beta_{h m} \theta_{1} I_{m}\right) S_{h}-\left(\theta_{2}+\mu_{h}+u_{1}\right) I_{h}\right)+\lambda_{z_{h}}\left(\left(\theta_{2}+\mu_{h}\right) I_{h}-\left(\gamma_{h}+u_{2}\right) Z_{h}\right)+ \\
& \left.\lambda_{R_{h}}\left(\gamma_{h} Z_{\hat{h}}-\gamma_{1 h} R_{h}+u_{1} I_{h}+u_{2} Z_{\hbar h}\right)+\lambda_{S_{m}}\left(B_{m}-\left(\beta_{m h} \theta_{1} I_{h}\right) S_{m}\right)+\lambda_{I_{h}}\left(\left(\beta_{h m} \theta_{1} I_{h}\right) S_{m}-\left(\theta_{2}\right) I_{m}\right)+\lambda_{z_{m}}\left(\left(\theta_{2}\right) I_{h}-\left(\gamma_{m}\right) Z_{\hbar h}\right)\right]
\end{aligned}
$$

And $\lambda_{S_{h}}^{s}=-\left[\lambda_{S_{h}}\left(-\left(\beta_{h m} \theta_{1} I_{m}-\phi_{h}\right)+\lambda_{I_{h}} \beta_{h m} \theta_{1} I_{m}\right)\right]$

$\lambda_{I_{h}}^{r}=$

$\left[A_{1}+c_{1} u_{1}+\lambda_{S_{h}}\left(-\left(\beta_{h m} \theta_{1}\right) S_{h}+\right.\right.$

$\lambda_{u_{h}}\left(\left(\beta_{h m} \theta_{1}\right) S_{h}-\left(\theta_{2}+\mu_{h}+u_{1}\right)\right)$

$\left.+\lambda_{z_{h}}\left(\theta_{2}+\mu_{h}\right)+\lambda_{R_{h}}\left(u_{1}\right)\right]$

$\lambda_{z_{h}}^{s}=-\left[A_{2}+c_{2} u_{2}+\lambda_{z_{h}}\left(-\left(\gamma_{h}+u_{2}\right)\right)+\lambda_{\pi_{h}}\left(\gamma_{h}+u_{2}\right)\right]$

and

$\lambda_{\mathbb{R}_{h}}^{r}=-\left[\lambda_{S_{h}} \gamma_{1 \hbar}+\lambda_{R_{h}}\left(-\gamma_{1 h}\right)\right]$

$\lambda_{S_{m}}^{s}=-\left[\lambda_{s_{m}}\left(-\left(\beta_{m n} \theta_{1} I_{n h}\right)+\lambda_{I_{m}} \beta_{m n} \theta_{1} I_{n}\right)\right]$

$\lambda_{I_{m}}^{r}=-\left[\lambda_{S_{m}}\left(-\left(\beta_{m h} \theta_{1}\right) S_{m}+\lambda_{I_{m}}\left(\left(\beta_{m h} \theta_{1}\right) S_{m}-\left(\theta_{2}\right)\right)\right.\right.$

$\left.+\lambda_{z_{m}}\left(\theta_{2}\right)\right]$

$\lambda_{z_{h}}^{s}=-\left[\lambda_{z_{h}}\left(-y_{h}\right)\right](18)$

With transversality conditions

$\lambda_{S_{h}}(T)=\lambda_{I_{h}}(T)=\lambda_{z_{h}}(T)=\lambda_{R_{h}}(T)=\lambda_{S_{m}}(T)=\lambda_{h_{m}}(T)=\lambda$

The optimal control $u_{1}^{*}$ and $u_{2}^{*}$ is given by

$$
\begin{array}{r}
u_{1}^{*}=\min \left\{\max \left(0, \frac{1}{w_{1}}\left(\lambda_{I}-\lambda_{R}-c_{1}\right) I_{s} 1\right\}\right. \\
u_{2}^{*}=\min \left\{\max \left(0, \frac{1}{w_{2}}\left(\lambda_{z}-\lambda_{R}-c_{2}\right) Z_{s} 1\right\}\right.
\end{array}
$$

\section{Numerical Illustration}

In the simulation we consider various parameter values and some hypothetical values for state variables. First, we considered the model without any control measures and compared the state variables. Fig. 3 (a-d) gives a comparison of state variables when the loss of immunity is considered and the optimal controls are not in operation. Fig.3 (c-d) gives the effect of lost immunity on the state variables. The number of infectives (symptomatic) is more when lost immunity is considered $\left(\gamma_{1}>0\right)$ as compared to that when lost immunity is not considered in the model $\left(\gamma_{1}=0\right)$. Fig. 4 illustrates the comparison of the effect of lost immunity on removals (immunized or recovery ).

We study the effect of controls on the number of individuals of asymptomatic and symptomatic considering effect of eof 6 trb (Fig. 5 (a-c)). Fig.5(c) illustrates that treating asymptomatic individuals alone will be more than treating the symptomatic individuals.

Table: Model parameter values. Figures in parenthesis are the values used for estimating state variables.

\begin{tabular}{|c|c|c|c|}
\hline Parameter & Description & Value & Reference \\
\hline$B_{h m}$ & Recruitment of susceptible & $(1 / 60)$ X365 & $(10)$ \\
\hline$\beta_{h m}$ & Contact rate between bacterial and susceptible hosts & $0.03-0.5(\mathbf{0 . 4})$ & $(1,7,13)$ \\
\hline$\theta_{1}$ & Proportion of infected individuals who are infectious & $0.15-0.6(\mathbf{0 . 6})$ & $(11)$ \\
\hline$\theta_{2}$ & Proportion of infected mosquitoes who are also infectious & $0.02-0.5(\mathbf{0 . 0 2 8})$ & $(11)$ \\
\hline$\mu_{h}$ & Immigration of infectious individuals & 0.00004 & $(4)$ \\
\hline$\emptyset_{h}$ & Rate at which susceptible remain uninfected even after contract & $0.001-0.03(\mathbf{0 . 0 2 8})$ & Assumed \\
\hline$\gamma_{h}$ & Rate at which individuals acquired immunity & 0.0146 & $(4)$ \\
\hline$\gamma_{1}$ & Rate at which removed individuals lose their immunity & $0.001-0.01(\mathbf{0 . 0 1})$ & Assumed \\
\hline
\end{tabular}

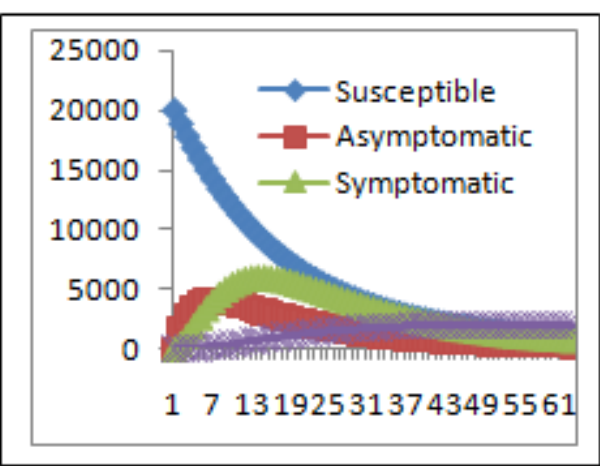

(a)

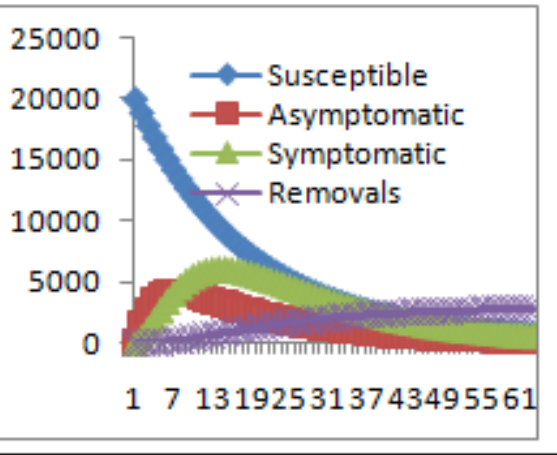

(b)

(a) $\left(\gamma_{1}=0\right.$ and Time independent immunity $)$

(b) $\left(\gamma_{1}=0\right.$ and Time dependent immunity)

Volume 4 Issue 12, December 2015 
International Journal of Science and Research (IJSR)

ISSN (Online): 2319-7064

Index Copernicus Value (2013): 6.14 | Impact Factor (2014): 5.611

(c) $\left(\gamma_{1}>0\right.$ and Time independent immunity)

(d) $\left(\gamma_{1}>0\right.$ and Time dependent immunity)

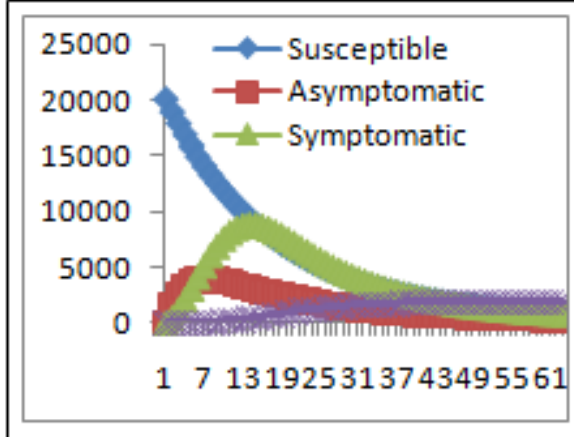

(c)

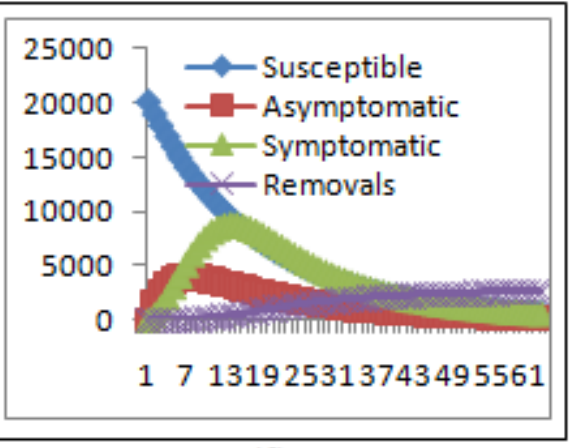

(d)

Figure 3: Effect of lost immunity on the state variables (a-d)

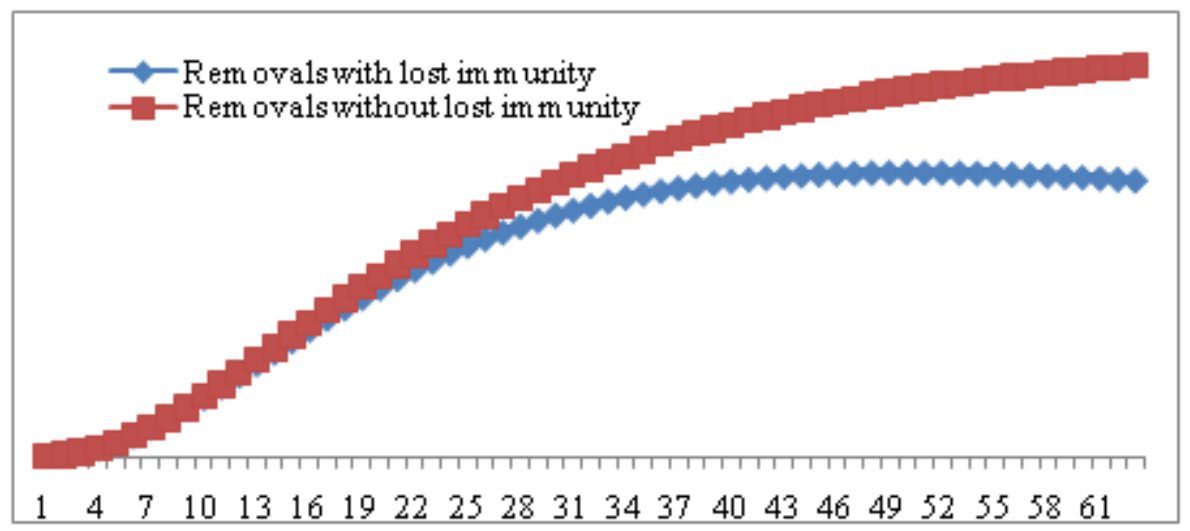

Figure 4: Comparison of removals with and without lost immunity

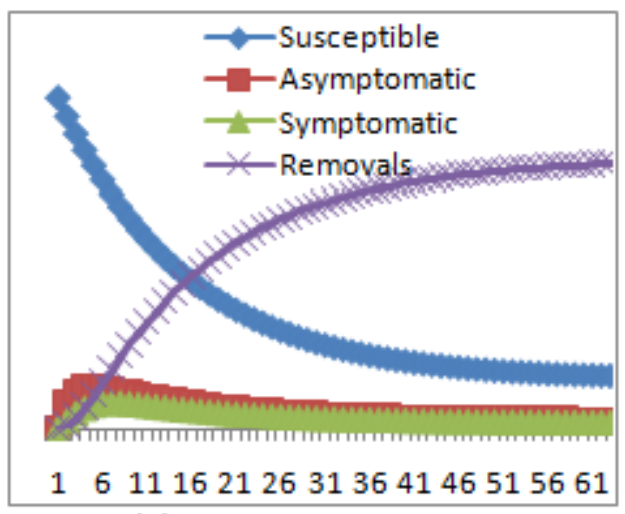

(a) $\left(u_{1} \neq 0\right.$ and $\left.u_{2} \neq 0\right)$

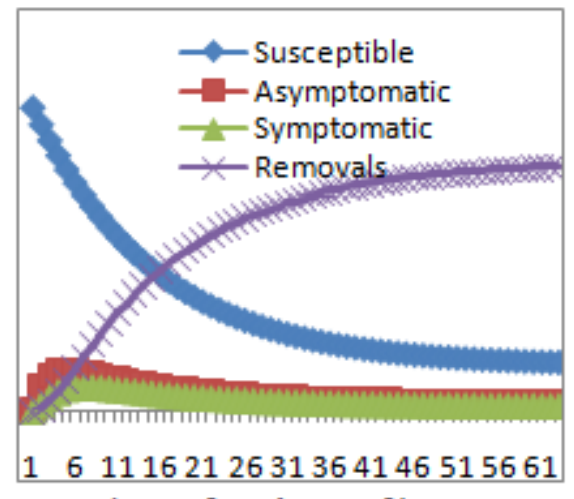

(b) $\left(u_{1}=0\right.$ and $\left.u_{2} \neq 0\right)$

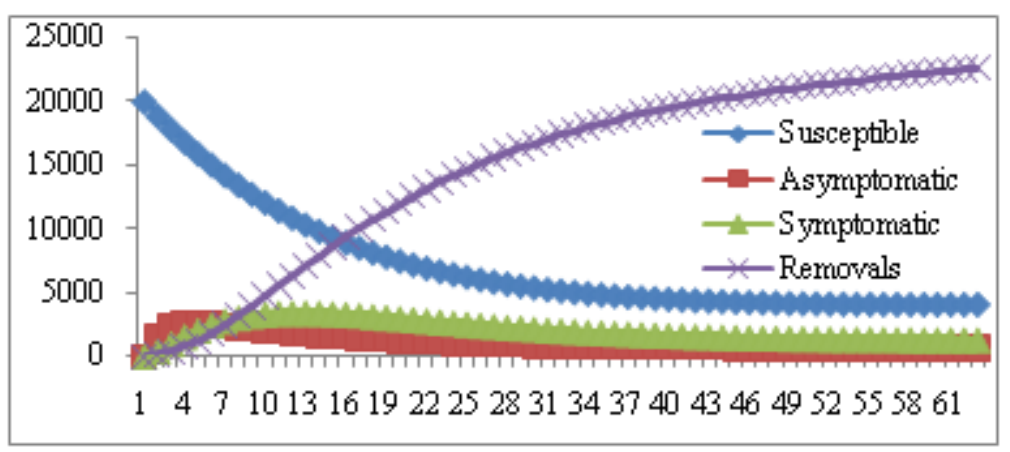

(c) $\left(u_{1} \neq 0\right.$ and $\left.u_{2}=0\right)$

Figure 5: Optimal controls (a-c)

\section{Volume 4 Issue 12, December 2015}




\section{References}

[1] Agusto, F. B., Marcus, N. and Okosum K. O., (2012): Application of optimal control to the epidemiology of malaria. Electronic Journal of Differential Equations, 81, 1-22.

[2] Anderson, R. M. and May, R. M. (2005): Directly transmitted infectious diseases: control by vaccination. Science, 215, 1053-1060.

[3] Aron, J. L. and May, R. M., (1982): The population dynamics of malaria, in R. M. Anderson, ed., Population dynamics of infectious disease. Chapman and Hall, London, 139-179.

[4] Cushing, C. J. and Hyman, J., (2006): Bifurcation analysis of mathematical model for malaria transmission. SIAM Journal of Applied Mathematics, 67(1), 24-25.

[5] Hethcote, H. W., (2000): The mathematics of infectious diseases. SIAM Rev., 42(4).

[6] Macdonald, G., (1957): The epidemiology and control of malaria. London, Oxford University Press.

[7] Mukandavire, C., musuk, G., Magombedze, G. and Mukandavire, Z., (2010): Malaria model with immigrantion of infectives and seasonal forcing in transmission. International Journal of Applied Mathematics and Computation, 2.

[8] Mwanga, G. G. , Haario, H. and Nannyonga, K., (2014a) : Optimal control of antimalarial drug resistance with infective immigrants. International Journal of Biomathematics.

[9] Mwanga, G. G. , Haario, H. and Nannyonga, K., (2014b) : Optimal control of of malaria model with antimalarial drug resistance in the presence of parameter uncertainty. Applied Mathematical sciences, 8(55), 2701-2730.

[10] Nedelman, J., (1985): Estimation for a model of multiple malaria infections. Biometrics, 41(2), 447-453.

[11] Ross, R., (1915): Some a priori pathometric equations. British medical Journal. 1 (2830), 546.

[12] Smith, D. L. and McKenzie, F. ES., (2004): Statics and dynamics of malaria infection in Anopheles mosquitoes. Malaria Journal, 3.

[13] Smith, D.L., Battle, K. E., Hay, S.L., Barker, C. M., Scott, T. W. and McKenzie, F. E., (2012): Ross, Macdonald and a theory for the dynamics and control of mosquito-transmitted pathogens. PLoS Pathogens 8 (4), e1002588.

[14] Talawar, A. S. and Pujar, H. S. (2011): Epidemiological situation of malaria in irrigated areas: A case study of Raichur district, Karnataka. KJAS, 24(2), 198-200. 